\title{
Second Order Local Analysis for 3D Reconstruction of Specular Surfaces
}

\author{
Silvio Savarese, Min Chen and Pietro Perona \\ California Institute of Technology \\ Mail stop 136-93, Pasadena, CA 91125 \\ \{savarese,perona\}@vision.caltech.edu \\ chen@cs.caltech.edu
}

\begin{abstract}
We analyze the problem of recovering the shape of a mirror surface. A calibrated scene composed of lines passing through a point is assumed. The lines are reflected by the mirror surface onto the image plane of a calibrated camera, where the intersection, orientation and curvature of such reflections are measured. The relationship between the local geometry of the surface around the point of reflection and the measurements is analyzed. We extend the analysis in [13, 14], where we recovered positions and normals and second order local geometry of a specular surface up to one unknown parameter. We show that, provided that we work in a neighborhood of a surface whose third order surface terms can be neglected, the second order parameter ambiguity can be solved by equating the curvatures observed for the reflected lines with those computed from analytical differentiation followed by a perspective projection.
\end{abstract}

\section{Introduction and Motivation}

We are interested in the possibility of recovering information on the shape of a surface from the specular component of its reflectance function. Since we wish to ignore the contributions of shading and texture, we will study surfaces that are perfect mirrors. A curved mirror surface produces 'distorted' images of the surrounding world. For example, the image of a straight line reflected by a curved mirror is, in general, a curve.
It is clear that such distortions are systematically related to the shape of the surface. In the previous work $[13,14]$, we presented a novel study on the basic geometrical principles relating the shape of a mirror surface to the distorted scene it produces. We assumed a calibrated world composed of the simplest primary structures: one point and one or more lines through it. We studied the relationship between the position and local geometry of the mirror surface around the point of reflection, and the orientation and curvature of the reflected images of such point and lines. We showed that it is possible to recover positions and normals and partial second order surface geometry. In fact, the local second order description of the surface can be explicitly expressed up to one unknown parameter. In this paper, we extend the analysis by showing that such a parameter can be estimated by equating the curvatures observed for the reflected lines with those computed from second order analytical differentiation followed by a perspective projection. Such a conclusion is valid in a neighborhood around the reflection point where the third order terms of the surface may be neglected.

Applications of our work include recovering and inspecting objects with highly glossy surfaces, such as cars, industrial parts, etc... Additionally, our work may provide useful mathematical tools for analyzing and calibrating omniview cameras with curved mirror surfaces.

In Sec. 2 we briefly review the first order analysis addressed in our previous work $[13,14]$. Then, we extend it to the second order analysis in Sec. 3. Finally, we conclude the paper with a discussion on our find- 
ings and a number of issues for further investigation.

\subsection{Related Work}

Many researchers have used highlights as a cue to infer information about the geometry of a specular surface. Koenderink and van Doorn [10] qualitatively described how pattern of specularities changes under viewer motion. This analysis was extended by Blake et al. and incorporated in a stereoscopic vision framework [3, 2]. Additionally, Zisserman et al. [16] investigated what geometrical information can be obtained from tracked motion of specularities. Some approaches were based on mathematical models of specular reflections (e.g. reflectance maps) [8] or extension of photometric stereo models [9]. Oren and Nayar [11] performed a classification of real and virtual features, and developed an algorithm recovering the 3D surface profiles traveled by virtual features. Zheng and Murata [15] developed a system where a rotating specular object is reconstructed by illuminating it by extended lights and then analyzing the motion of the highlight stripes. Apart from these efforts on local analysis, some authors $[7,12]$ have also addressed the issue of reconstructing a specular surface globally. For instance, Halsead et al. [7] proposed a reconstruction algorithm where a surface global model is fitted to a set of normals by imaging a pattern of light reflected by specular surface. Their results were applied to the interactive visualization of the cornea.

Our method departs from these previous techniques in several aspects. First, surrounding world and viewer are assumed to be static. Second, monocular images rather than stereo pairs are needed for the reconstruction. Finally, our analysis is local and differential rather than global and algebraic.

\section{First Order Analysis}

Our goal is to obtain local geometrical information about an unknown smooth mirror surface, up to the second order. Previously, we proposed a first-order analysis to obtain a mathematical constraint given by a pattern line and its observed reflected curve in the image plane, which allows us to estimate first and second surface parameters, up to one parameter ambiguity. The focus of this paper is to get rid of this ambiguity
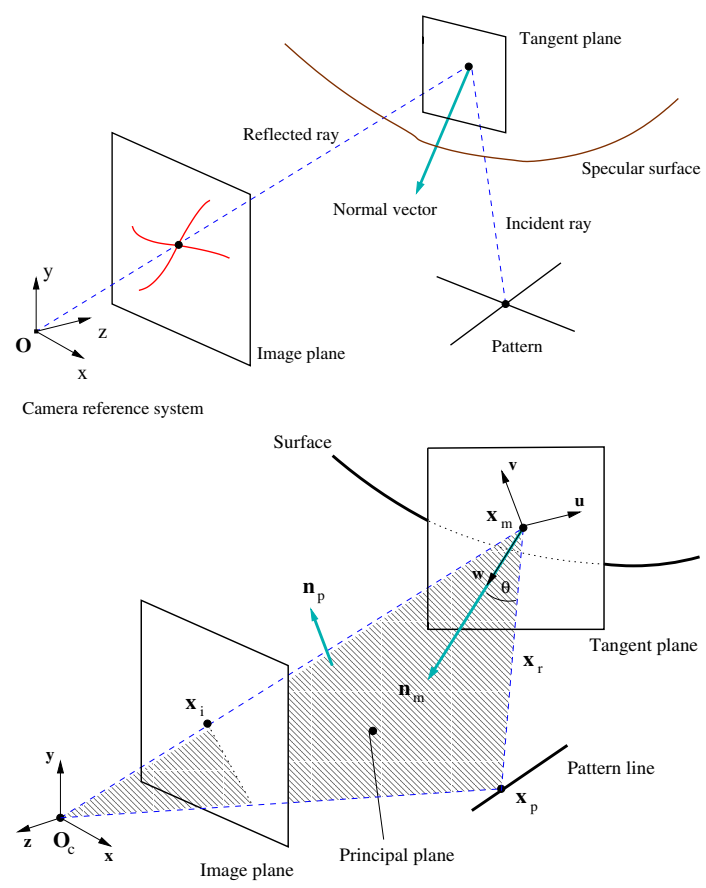

Figure 1. Upper panel: the basic setup. Lower panel: the geometry.

using a higher order analysis. Before we start to elaborate the second-order analysis in Sec. 3, we would like to briefly summarize the major results from our previous work, and detailed derivations can be found in $[13,14]$.

\subsection{Experimental Setup and Notations}

The basic geometric setup is depicted in Fig. 1 (upper panel). A calibrated pattern is reflected by a curved mirror surface and then observed by a calibrated camera. By default, a point (or a vector) in the 3D space is expressed as a column 3 -vector and is denoted by a bold letter (e.g. $\left.\mathbf{x}=(x y z)^{T}\right)$. A unit vector is denoted by a bold letter with hat (e.g. $\hat{\mathbf{n}}$ ).

A coordinate reference system $[X Y Z]$ is chosen with its origin located at $\mathbf{O}_{c}$, the center of projection of the camera. Let $\mathbf{x}_{p}$ be the pattern point, we use $\mathbf{x}_{i}$ to denote the image of $\mathbf{x}_{p}$ reflected by the surface and $\mathbf{x}_{m}$ to denote the corresponding reflection point on the mirror surface. Since the camera and pattern are calibrated, $\mathbf{x}_{p}$ and $\mathbf{x}_{i}$ are known, whereas $\mathbf{x}_{m}$ is unknown. The normal to the surface at $\mathbf{x}_{m}$ is indicated by $\hat{\mathbf{n}}_{m}$ 
and is unknown as well. Let us call the plane defined by $\mathbf{x}_{i}, \mathbf{x}_{p}$ and $\mathbf{O}_{c}$ the principal plane and let $\hat{\mathbf{n}}_{p}$ be its normal vector. Hence $\hat{\mathbf{n}}_{p}$ is a known quantity. Let $s$ be the distance between the center of the camera $\mathbf{O}_{c}$ and $\mathbf{x}_{m}$, we have shown that $\mathbf{x}_{m}$, the normal $\mathbf{n}_{m}$ and reflection angle $\theta$ can be completely determined by this single parameter as follows [13]:

$$
\begin{aligned}
\mathbf{x}_{m} & =s \mathbf{x}_{i}, \\
\mathbf{n}_{m} & =\left[\hat{\mathbf{x}}_{i}-\left(s \hat{\mathbf{x}}_{i}-\mathbf{x}_{p}\right) /\left\|s \hat{\mathbf{x}}_{i}-\mathbf{x}_{p}\right\|\right] \times \hat{\mathbf{n}}_{p},(1) \\
\cos \theta & =\frac{\sqrt{2}}{2} \sqrt{\frac{s-\hat{\mathbf{x}}_{i}^{T} \mathbf{x}_{p}}{\left\|s \hat{\mathbf{x}}_{i}-\mathbf{x}_{p}\right\|}+1} .
\end{aligned}
$$

Therefore, recovering the local geometry of the mirror surface up to the first order accuracy boils down to determining $s$.

In this paper we actually work in a more suitable coordinate reference system $[U V W]$, called principal reference system (see Fig. 1 - lower panel), which was first introduced by Blake [2]. The principal reference system is centered in $\mathbf{x}_{m}$ : the $\hat{\mathbf{w}}$ axis is coincident with $\hat{\mathbf{n}}_{m}(s)$, the $\hat{\mathbf{v}}$ axis is coincident with $\hat{\mathbf{n}}_{p}$, and the $\hat{\mathbf{u}}$ axis is given by $\hat{\mathbf{u}}=\hat{\mathbf{v}} \times \hat{\mathbf{w}}$.

Since we are interested in analyzing the surface locally, we can consider the corresponding Monge representation of the surface; that is, the surface can be described by a graph $z=G(x, y)$. In the principal reference system, the normal of the surface at the origin is $\hat{\mathbf{w}}$ and the tangent plane to the surface at the origin is the plane defined by $\hat{\mathbf{u}}$ and $\hat{\mathbf{v}}$. Therefore we may express the surface around $\mathbf{x}_{m}$ implicitly from its special Monge form [6], that is

$$
\begin{aligned}
g(u, v, w)= & w-\frac{1}{2}\left(a u^{2}+2 c u v+b v^{2}\right)- \\
& \frac{1}{6}\left(e u^{3}+3 f u^{2} v+3 g u v^{2}+h v^{3}\right)+\cdots,
\end{aligned}
$$

where $a, b, c, \cdots$ are higher-order surface parameters to be recovered.

\subsection{The Tangent Line Constraint}

To estimate the location $s$ of $\mathbf{x}_{m}$ and the secondorder surface parameters $a, b, c$, we consider a pattern line through $\mathbf{x}_{p}$, oriented in the direction of $\Delta \mathbf{p}=$ $\left[\begin{array}{lll}\Delta p_{u} & \Delta p_{v} & \Delta p_{w}\end{array}\right]^{T}$ and parameterized by $t$, that is, $\mathbf{x}_{p}(t)=\mathbf{x}_{p o}+t \boldsymbol{\Delta} \mathbf{p}$. Given a fixed observer $\mathbf{O}_{c}$ and this parameterized pattern line, we may define a function $\mathbf{f}: t \in \Re \rightarrow \mathbf{x}_{m} \in \Re^{3}$, mapping from a point $\mathbf{x}_{p}$ on the line to its corresponding reflection point $\mathbf{x}_{m}$ in the mirror surface. In other words, $\mathbf{f}$ defines a parameterized space curve $\mathbf{f}(t)$ on the mirror surface, which specifies how the position of the reflection point $\mathbf{x}_{m}$ changes as $t$ varies. When $t=t_{o}=0, \mathbf{x}_{m}=$ $\mathbf{x}_{m_{o}}=\mathbf{f}\left(t_{o}\right)$, namely, the origin of the principal reference system. The pattern line, reflected by the mirror surface, is imaged as a curve in the image plane. We call such a curve $\mathbf{f}_{i}(t)$, which is essentially the perspective projection of $\mathbf{f}(t)$ into the image plane. Let $\hat{\mathbf{t}}_{o}=\left[\begin{array}{lll}\dot{u}(0) & \dot{v}(0) & \dot{w}(0)\end{array}\right]^{T}$ and $\hat{\mathbf{t}}_{i o}$ be the tangent vectors of the curves $\mathbf{f}(t)$ and $\mathbf{f}_{i}(t)$ at $t_{o}$ respectively. Then $\hat{\mathbf{t}}_{i o}$ and $\hat{\mathbf{t}}_{o}$ are linked by the following relationship:

$$
\hat{\mathbf{t}}_{o}=\hat{\mathbf{n}}_{m} \times\left(\hat{\mathbf{O}}_{c} \times \hat{\mathbf{t}}_{i o}\right) /\left\|\hat{\mathbf{n}}_{m} \times\left(\hat{\mathbf{O}}_{c} \times \hat{\mathbf{t}}_{i o}\right)\right\| .
$$

Thus, with $\hat{\mathbf{t}}_{i o}$ measured, $\hat{\mathbf{t}}_{o}$ is known, up to $s$.

We now present the main contribution from our previous work: an analytical formula for $\hat{\mathbf{t}}_{o}$ from firstorder analysis. Inspired by the work of Chen and Arvo [5], by applying Fermat principle [4] and Lagrange Multiplier Theorem, we obtain a Fermat equation $F\left(t, \mathbf{x}_{m}, \lambda\right)=\mathbf{0}$ satisfied by the observed specular reflection, which has an explicit form as follows:

$$
\begin{aligned}
\hat{\mathbf{x}}_{r}\left(\mathbf{x}_{m}, t\right)+\hat{\mathbf{x}}_{i}\left(\mathbf{x}_{m}\right)+\lambda \nabla g\left(\mathbf{x}_{m}\right) & =\mathbf{0}, \\
g\left(\mathbf{x}_{m}\right) & =0,
\end{aligned}
$$

where $g$ is given in equation (2). It then follows from the Implicit Function Theorem that we may compute the derivative of the smooth mapping function $\mathbf{f}$ with respect to $t$ by

$$
\mathbf{B}\left(t, \mathbf{x}_{m}, \lambda\right)=-\mathbf{J}\left(t, \mathbf{x}_{m}, \lambda\right) \mathbf{M}(t),
$$

where

$$
\begin{aligned}
\mathbf{B} & =\frac{\partial F}{\partial t}=\left[\begin{array}{c}
\mathbf{B}_{31} \\
0
\end{array}\right], \\
\mathbf{J} & =\frac{\partial F}{\partial\left(\mathbf{x}_{m}, \lambda\right)}=\left[\begin{array}{cc}
\mathbf{J}_{33} & \nabla g\left(\mathbf{x}_{m}\right) \\
\left(\nabla g\left(\mathbf{x}_{m}\right)\right)^{\mathrm{T}} & 0
\end{array}\right](6) \\
\mathbf{M}(t) & =\left[\begin{array}{ll}
\dot{u}(t) \dot{v}(t) \dot{w}(t) \dot{\lambda}(t)]^{T}=[\dot{\mathbf{f}}(t) \dot{\lambda}(t)
\end{array}\right]^{T} .
\end{aligned}
$$

Here the submatrices $\mathbf{B}_{31}$ and $\mathbf{J}_{33}$ (the subscript denotes the matrix dimension) are defined as

$$
\begin{aligned}
\mathbf{B}_{31} & =-\frac{\left(\mathbf{I}-\hat{\mathbf{x}}_{r} \hat{\mathbf{x}}_{r}^{\mathrm{T}}\right) \Delta \mathbf{p}}{\left\|\mathbf{x}_{m}-\mathbf{x}_{p}(t)\right\|}, \\
\mathbf{J}_{33} & =\frac{\left(\mathbf{I}-\hat{\mathbf{x}}_{r} \hat{\mathbf{x}}_{r}^{\mathrm{T}}\right)}{\left\|\mathbf{x}_{m}-\mathbf{x}_{p}(t)\right\|}+\frac{\left(\mathbf{I}-\hat{\mathbf{x}}_{i} \hat{\mathbf{x}}_{i}^{\mathrm{T}}\right)}{\left\|\mathbf{x}_{m}-O_{c}\right\|}+\lambda \mathbf{H}_{g} .
\end{aligned}
$$


If the third-order terms are negligible, it follows from equation (2) that the gradient vector $\nabla g$ and the Hessian matrix $\mathbf{H}_{g}$ of $g$ are given respectively by

$\nabla g=\left[\begin{array}{c}-a u-c v \\ -c u-b v \\ 1\end{array}\right], \quad \mathbf{H}_{g}=\left[\begin{array}{ccc}-a & -c & 0 \\ -c & -b & 0 \\ 0 & 0 & 0\end{array}\right]$

By evaluating equation (5) at the known path $\left(\mathbf{x}_{p_{0}}, \mathbf{x}_{m_{0}}, O_{c}\right)$ and then solving for $\dot{\mathbf{f}}(0)$, we obtain that $\dot{w}(0)=0$ and $\left[\begin{array}{lll}\dot{u}(0) & \dot{v}(0) & \dot{\lambda}(0)\end{array}\right]^{\mathrm{T}}$ is given by

$-\frac{1}{\Delta}\left[\begin{array}{ccc}J_{v}-2 b \cos \theta & 2 c \cos \theta & 0 \\ 2 c \cos \theta & J_{u}-2 a \cos \theta & 0 \\ -J_{w}\left(J_{v}-2 b \cos \theta\right) & -2 c \cos \theta J_{w} & \Delta\end{array}\right]\left[\begin{array}{c}B_{u} \\ B_{v} \\ B_{w}\end{array}\right]$

where

$$
\begin{aligned}
\Delta & =\left(J_{u}-2 a \cos \theta\right)\left(J_{v}-2 b \cos \theta\right)-4 c^{2} \cos ^{2} \theta \\
B_{u} & =\left(\Delta \mathbf{p}_{w} \cos \theta \sin \theta-\Delta \mathbf{p}_{u} \cos ^{2} \theta\right) /\left\|\mathbf{x}_{p_{0}}\right\|, \\
B_{v} & =-\Delta \mathbf{p}_{v} /\left\|\mathbf{x}_{p_{0}}\right\|, \\
B_{w} & =\left(\Delta \mathbf{p}_{u} \cos \theta \sin \theta-\Delta \mathbf{p}_{w} \cos ^{2} \theta\right) /\left\|\mathbf{x}_{p_{0}}\right\|, \\
J_{v} & =\left(s+\left\|\mathbf{x}_{p_{0}}\right\|\right) /\left(s\left\|\mathbf{x}_{p_{0}}\right\|\right), \quad J_{u}=J_{v} \cos ^{2} \theta, \\
J_{w} & =\left(\left(\left\|\mathbf{x}_{p_{0}}\right\|-s\right) \sin \theta \cos \theta\right) /\left(s\left\|\mathbf{x}_{p_{0}}\right\|\right)
\end{aligned}
$$

Let $\psi$ be the angle between $\hat{\mathbf{t}}_{o}$ and $\hat{\mathbf{u}}$ computed using equation (3) from the measurement of $\hat{\mathbf{t}}_{i_{0}}$, we may impose the following constraint on $a, b, c$ and $s$ :

$\tan \psi=\frac{\dot{v}(0)}{\dot{u}(0)}=\frac{\left(J_{u}-2 a \cos \theta\right) B_{v}+2 c B_{u} \cos \theta}{\left(J_{v}-2 b \cos \theta\right) B_{u}+2 c B_{v} \cos \theta}$.

\subsection{Recovering Second-Order Surface Parameters}

By examining three or more pattern lines, equation (9) suggests a linear system satisfied by $a, b, c$, denoted by a matrix $\mathbf{H}(s)$ depending on $s$. By analyzing the properties of $\mathbf{H}$, the authors [14] have demonstrated: 1) the distance parameter $s$ of the unknown surface can be determined by solving $\operatorname{det}\left(\mathbf{H}^{\mathrm{T}} \mathbf{H}\right)=0$. 2) given $s$, the second order parameters $a, b, c$ of the unknown specular surface can be recovered as functions of a single parameter $k$, which may be determined by performing a second-order analysis.

\section{Second Order Analysis}

Given a pattern line, we perform this higher order analysis by comparing the curvature measured for the observed reflection curve in the image plane with that computed from analytical differentiation followed by a perspective projection. In detail, the basic procedure can be stated in four steps:

Step 1: (Second Order Differentiation)

By differentiating equation (5) with respect to $t$, we have

$$
\frac{d \mathbf{B}\left(t, \mathbf{x}_{m}, \lambda\right)}{d t}=-\frac{d \mathbf{J}\left(t, \mathbf{x}_{m}, \lambda\right)}{d t} \mathbf{M}-\mathbf{J} \frac{d \mathbf{M}(t)}{d t},
$$

where $\frac{d \mathbf{M}}{d t}=\left[\begin{array}{lll}\ddot{\mathbf{f}}(t) & \ddot{\lambda}(t)\end{array}\right]^{\mathrm{T}}=\left[\begin{array}{llll}\ddot{u}(t) & \ddot{v}(t) & \ddot{w}(t) & \ddot{\lambda}(t)\end{array}\right]^{\mathrm{T}}$. Our goal is to compute $\ddot{\mathbf{f}}(0)$. For this purpose, we are required to compute the components $d \mathbf{B} / d t$ and $d \mathbf{J} / d t$ in equation (10). Note that we should also consider $\left(\mathbf{x}_{m}, \lambda\right)$ as a function of $t$ (i.e., the function $\mathbf{f}$ defined in Sec. 2.2) while calculating these derivatives. This is also the reason that we used the notation $d / d t$ instead of $\partial / \partial t$. Due to space limit, we only sketch some major formulae here, and the detailed derivation can be seen in our forthcoming journal paper. Let $N_{r}=\mathbf{I}-\hat{\mathbf{x}}_{r} \hat{\mathbf{x}}_{r}^{\mathrm{T}}$ and $N_{i}=\mathbf{I}-\hat{\mathbf{x}}_{i} \hat{\mathbf{x}}_{i}^{\mathrm{T}}$. By defining

$$
A=\frac{N_{r} \hat{\mathbf{x}}_{r}^{\mathrm{T}} \Delta \mathbf{p}+N_{r} \Delta \mathbf{p} \hat{\mathbf{x}}_{r}^{\mathrm{T}}+\hat{\mathbf{x}}_{r} \Delta \mathbf{p}^{\mathrm{T}} N_{r}}{\left\|\mathbf{x}_{m}-\mathbf{x}_{p}(t)\right\|^{2}}
$$

we can derive that

$$
\frac{\partial \mathbf{B}_{31}}{\partial t}=-A \Delta \mathbf{p}, \quad \frac{\partial \mathbf{B}_{31}}{\partial \mathbf{x}_{m}}=A .
$$

Consequently, the application of the chain rule yields

$$
\frac{d \mathbf{B}\left(t, \mathbf{x}_{m}, \lambda\right)}{d t}=\left[\begin{array}{c}
A(\Delta \mathbf{p}+\dot{\mathbf{f}}(t)) \\
0
\end{array}\right] .
$$

Similarly, we can compute $d \mathbf{J} / d t$ through $\partial \mathbf{J} / \partial t$ and $\partial \mathbf{J} / \partial\left(\mathbf{x}_{m}, \lambda\right)$. It is easy to compute that

$$
\frac{\partial \mathbf{J}}{\partial t}=\left[\begin{array}{cc}
\partial \mathbf{J}_{33} / \partial t & 0 \\
0 & 0
\end{array}\right]=\left[\begin{array}{cc}
A & 0 \\
0 & 0
\end{array}\right] .
$$

The computation of $\partial \mathbf{J} / \partial\left(\mathbf{x}_{m}, \lambda\right)$ becomes a little more complicated, since the result turns out to be a third order tensor, which, however, after multiplied by M, still returns a matrix. For clarity, we view this third order tensor as stacks of matrices, each corresponding to the differential matrix associated with a column of $\mathbf{J}$. Specifically, let $\mathbf{e}_{1}=(1,0,0)^{\mathrm{T}}, \mathbf{e}_{2}=(0,1,0)^{\mathrm{T}}, \mathbf{e}_{3}=$ 
$(0,0,1)^{\mathrm{T}}$, we may express the $j$ th column of $\mathbf{J}_{33}$, denoted by $\mathbf{J}_{33}^{j}$, as

$$
\mathbf{J}_{33}^{j}=\frac{N_{r} \mathbf{e}_{j}}{\left\|\mathbf{x}_{m}-\mathbf{x}_{p}(t)\right\|}+\frac{N_{i} \mathbf{e}_{j}}{\left\|\mathbf{x}_{m}-O_{c}\right\|}+\lambda \mathbf{H}_{g}^{j}
$$

Here and throughout the paper, we always use superscripts to denote the matrix column. By analogy with computing $\partial \mathbf{B}_{31} / \partial \mathbf{x}_{m}$, we have

$$
\begin{aligned}
T_{r}^{j} & =\partial\left(N_{r} \mathbf{e}_{j} /\left\|\mathbf{x}_{m}-\mathbf{x}_{p}(t)\right\|\right) / \partial \mathbf{x}_{m} \\
& =-\frac{N_{r} \hat{\mathbf{x}}_{r}^{\mathrm{T}} \mathbf{e}_{j}+N_{r} \mathbf{e}_{j} \hat{\mathbf{x}}_{r}^{\mathrm{T}}+\hat{\mathbf{x}}_{r} \mathbf{e}_{j}^{\mathrm{T}} N_{r}}{\left\|\mathbf{x}_{m}-\mathbf{x}_{p}(t)\right\|^{2}} \\
T_{i}^{j} & =\partial\left(N_{i} \mathbf{e}_{j} /\left\|\mathbf{x}_{m}-O_{c}\right\|\right) / \partial \mathbf{x}_{m} \\
& =-\frac{N_{i} \hat{\mathbf{x}}_{i}^{\mathrm{T}} \mathbf{e}_{j}+N_{i} \mathbf{e}_{j} \hat{\mathbf{x}}_{i}^{\mathrm{T}}+\hat{\mathbf{x}}_{i} \mathbf{e}_{j}^{\mathrm{T}} N_{i}}{\left\|\mathbf{x}_{m}-O_{c}\right\|^{2}}
\end{aligned}
$$

When the third-order terms in the Monge form of the mirror surface are negligible, it is obvious from equation (8) that $\partial \mathbf{H}_{g}^{j} / \partial \mathbf{x}_{m}=0$. We may thus express $\partial \mathbf{J}_{33}^{j} / \partial\left(\mathbf{x}_{m}, \lambda\right)$ as a $3 \times 4$ matrix $\left[\begin{array}{ll}T_{r}^{j}+T_{i}^{j} & \mathbf{H}_{g}^{j}\end{array}\right]_{34}$. Moreover, using equation (8), we have $\partial(\nabla g) / \partial\left(\mathbf{x}_{m}, \lambda\right)=\left[\begin{array}{ll}\mathbf{H}_{g} & 0\end{array}\right]_{34}$. It then follows from equation (6) that

$$
\frac{\partial \mathbf{J}}{\partial\left(\mathbf{x}_{m}, \lambda\right)} \mathbf{M}=\left[\begin{array}{cc}
T & \mathbf{H}_{g} \dot{\mathbf{f}}(t) \\
\left(\mathbf{H}_{g} \dot{\mathbf{f}}(t)\right)^{\mathrm{T}} & 0
\end{array}\right],
$$

where $T$ is a $3 \times 3$ matrix with its $j$ th column of the form defined by $\left(T_{r}^{j}+T_{i}^{j}\right) \dot{\mathbf{f}}(t)+\mathbf{H}_{g}^{j} \dot{\lambda}(t)$. Combining equations (12) and (13), we may derive from the chain rule that

$$
\frac{d \mathbf{J}\left(t, \mathbf{x}_{m}, \lambda\right)}{d t}=\left[\begin{array}{cc}
A+T & \mathbf{H}_{g} \dot{\mathbf{f}}(t) \\
\left(\mathbf{H}_{g} \dot{\mathbf{f}}(t)\right)^{\mathrm{T}} & 0
\end{array}\right] .
$$

Finally, by substituting equations (11) and (14) into equation (10), we get

$$
\mathbf{D}=-\left[\begin{array}{cc}
\mathbf{J}_{33} & \nabla g\left(\mathbf{x}_{m}\right) \\
\left(\nabla g\left(\mathbf{x}_{m}\right)\right)^{\mathrm{T}} & 0
\end{array}\right]\left[\begin{array}{c}
\ddot{\mathbf{f}}(t) \\
\ddot{\lambda}(t)
\end{array}\right],
$$

where

$$
\mathbf{D}=\left[\begin{array}{c}
A(\Delta \mathbf{p}+\dot{\mathbf{f}}(t))+\left(A+T+\dot{\lambda}(t) \mathbf{H}_{g}\right) \dot{\mathbf{f}}(t) \\
\left(\mathbf{H}_{g} \dot{\mathbf{f}}(t)\right)^{\mathrm{T}} \dot{\mathbf{f}}(t)
\end{array}\right] .
$$

From the last row of equation (15), we attain

$$
a \dot{u}^{2}(t)+2 c \dot{u}(t) \dot{v}(t)+b \dot{v}^{2}(t)+\ddot{w}(t)=0 .
$$

Solving for $\ddot{w}(0)$ yields

$$
\ddot{w}(0)=-a \dot{u}^{2}(0)-2 c \dot{u}(0) \dot{v}(0)-b \dot{v}^{2}(0) .
$$

Accordingly, the first two rows of equation (15) give rise to $[\ddot{u}(0) \ddot{v}(0)]^{\mathrm{T}}$. That is,

$-\frac{1}{\Delta}\left[\begin{array}{cc}J_{v}-2 b \cos \theta & 2 c \cos \theta \\ 2 c \cos \theta & J_{u}-2 a \cos \theta\end{array}\right]\left[\begin{array}{c}D_{1}-J_{w} \ddot{w}(0) \\ D_{2}\end{array}\right]$,

where $D_{1}, D_{2}$ are the first two rows of $\mathbf{D}$. Note that all the components in equation (15) should be evaluated at the given path $\left(\mathbf{x}_{p_{0}}, \mathbf{x}_{m_{0}}, O_{c}\right)$, i.e. $t=0$.

Step 2: (Curvature of $\mathbf{f}$ at $\mathbf{x}_{m_{0}}$ )

With the first- and second-order derivative of $\mathbf{f}$ computed at $\mathbf{x}_{m_{0}}$, i.e., $\mathbf{f}(0)$ and $\ddot{\mathbf{f}}(0)$, the curvature of the reflection curve $\mathbf{f}(t)$ at $\mathbf{x}_{m_{0}}$ on the mirror surface can be computed according to the following identity:

$$
\kappa_{m_{0}}=\|\dot{\mathbf{f}}(0) \times \ddot{\mathbf{f}}(0)\| /\|\dot{\mathbf{f}}(0)\|^{3} .
$$

Step 3: (Curvature of $\mathbf{f}_{i}$ at $\mathbf{x}_{i_{0}}$ )

The curvature $\kappa_{m_{0}}$ on the mirror surface is closely related to the curvature $\kappa_{i_{0}}$ of the observed curve $\mathbf{f}_{i}(t)$ on the image plane through a perspective projection. It has been shown by Cipolla and Giblin [6] that they satisfy the following relationship

$\kappa_{i_{0}}=\left(s \kappa_{m_{0}}\left\langle O_{c}, \hat{\mathbf{b}}_{m_{0}}\right\rangle\right) /\left(1-\left\langle O_{c}, \hat{\mathbf{t}}_{m_{0}}\right\rangle^{2}\right)^{3 / 2}$,

where $\hat{\mathbf{t}}_{m_{0}}, \hat{\mathbf{b}}_{m_{0}}$ are the tangent vector and the binormal vector of the spatial curve $\mathbf{f}(t)$ at $\mathbf{x}_{m_{0}}$, respectively. Let $\hat{\mathbf{n}}_{\mathbf{f}_{0}}$ be the principal normal of $\mathbf{f}(t)$ at $\mathbf{x}_{m_{0}}$. It is known from differential geometry that

$$
\begin{aligned}
& \hat{\mathbf{t}}_{m_{0}}=\dot{\mathbf{f}}(0) /\|\dot{\mathbf{f}}(0)\|, \hat{\mathbf{b}}_{m_{0}}=\hat{\mathbf{t}}_{m_{0}} \times \hat{\mathbf{n}}_{\mathbf{f}_{0}}, \\
& \hat{\mathbf{n}}_{\mathbf{f}_{0}}=\left(\ddot{\mathbf{f}}(0)-\hat{\mathbf{t}}_{m_{0}}\left\langle\hat{\mathbf{t}}_{m_{0}}, \ddot{\mathbf{f}}(0)\right\rangle\right) /\left(\kappa_{m_{0}}\|\dot{\mathbf{f}}(0)\|^{2}\right) .
\end{aligned}
$$

Step 4: (Measuring Image Curvature $\kappa_{i_{0}}$ )

As the last step, by replacing the left hand side of equation (17) by the curvature measured for the observed reflection curve in the image plane, we get another constraint about the second order parameters $a, b, c$ of the unknown surface, because the previous steps have 
expressed the right hand side of equation (17) in terms of $a, b, c$. Besides, since $a, b, c$ depend on a single parameter $k$, this constraint actually constitutes an identity for determining $k$.

As we can see, measuring the image curvature corresponding to one pattern line is sufficient to estimate $k$, by just following the above 4 steps. However, usage of 2 or more lines (combined with corresponding curvature estimates) will allow us to make more robust estimation to reduce error from noise. With $k$ known, the parameters $a, b$ and $c$ are completely determined, and we have recovered the full second order representation of the unknown surface.

\section{Conclusion and Future Work}

We have presented an analysis for fully reconstructing the first- and second-order local parameters of a mirror surface. Assuming a calibrated world composed of one point and one or more lines through it, our analysis relates the position and local geometry of the mirror surface around the point of reflection, to the orientation and curvature of the reflected images of such point and lines. If only the first order information of these reflected images is considered (namely, the orientation of the reflected lines in the image plane), the second order surface geometry can be retrieved only up to one unknown parameter. Such an ambiguity can be resolved if the second order information (namely, the curvature of the reflected lines in the image plane) is also available. By comparing the measured curvatures with those computed from analytical differentiation, the unknown parameter can be estimated. Our analysis is valid in a neighborhood around the reflection point where the third order terms of the surface may be neglected.

Future work is needed to study how sensitive the estimated parameters are with respect to noise added to the tangents and curvature. We view our results as a promising start in the quest of computing the global shape of specular surfaces under fairly general conditions. The more interesting case of an uncalibrated world appears much more challenging and will most certainly require the integration of additional cues and some form of prior knowledge on the likely statistics of the scene geometry.

\section{References}

[1] T. Binford. Inferring surfaces from images. Artificial Intelligence, 17:205-244, 1981.

[2] A. Blake. Specular stereo. In IJCAI, pages 973-976, 1985.

[3] A. Blake and G. Brelstaff. Geometry from specularities. In Proc. of Int. Conf. of Computer Vision, pages 394-403, 1988.

[4] M. Born and E. Wolf. Principle of optics - Electromagnetic theory and propagation. OPergamon Press, 1965.

[5] M. Chen and J. Arvo. Theory and application of specular path perturbation. ACM Transactions on Graphics, 19:246-278, 2000.

[6] R. Cipolla and P. Giblin. Visual motion of curves and surfaces. Cambridge University Press, 2000.

[7] M. Halsead, A. Barsky, S. Klein, and R. Mandell. Reconstructing curved surfaces from reflection patterns using spline surface fitting normals. In SIGGRAPH, 1996.

[8] G. Healey and T. Binford. Local shape from specularity. Computer Vision, Graphics, and Image Processing, 42:62-86, 1988.

[9] K. Ikeuchi. Determining surface orientation of specular surfaces by using the photometric stereo method. IEEE Journal of Pattern Analysis and Machine Intelligence, 3:661-669, 1981.

[10] J. Koenderink and A. van Doorn. Photometric invariants related to solid shape. Optica Apta, 27:981-996, 1980.

[11] M. Oren and S. K.Nayar. A theory of specular surface geometry. Trans. International Journal of Computer Vision, pages 105-124, 1997.

[12] D. Perard. Automated Visual Inspection of Specular Surfaces with Structured-lighting Reflection Techiniques. PhD thesis, VDI Verlag (Nr. 869), 2001.

[13] S. Savarese and P. Perona. Local analysis for 3d reconstruction of specular surfaces. In Proc. of IEEE Conference of Computer Vision and Pattern Recognition., December 2001.

[14] S. Savarese and P. Perona. Local analysis for 3d reconstruction of specular surfaces - part ii. In Proc. of European Conference of Computer Vision. (Accepted), June 2002.

[15] J. Zheng and A. Murata. Acquiring a complete 3d model from specular motion under the illumination of circular-shaped light sources. IEEE Journal of Pattern Analysis and Machine Intelligence, 8, 2000.

[16] A. Zisserman, P. Giblin, and A. Blake. The information available to a moving observer from specularities. Image and Video Computing, 7:38-42, 1989. 